\title{
Role of Neural Activity during Synaptogenesis in Drosophila
}

\author{
Jill Jarecki ${ }^{1}$ and Haig Keshishian ${ }^{2}$ \\ Departments of ${ }^{1}$ Genetics and ${ }^{2}$ Biology, Yale University, New Haven, Connecticut 06511
}

This article reveals a novel aspect in the regulation of synaptic connectivity in Drosophila. Reducing neural activity genetically or pharmacologically disrupts the normally precise embryonic and larval neuromuscular connections. In third instar larvae with mutations that affect sodium channel function or expression such as no action potential, temperature-induced paralysis $E$, or seizure', foreign neuromuscular synapses, arising from inappropriate nerve sources, are observed on muscle fibers throughout the abdominal body wall. Their frequencies increase as neural activity is further reduced in double mutant combinations. These foreign connections are first observed during late embryogenesis as filopodial-like contacts, but critical period analysis suggests that neural activity must be reduced during both late embryogenesis and the first larval instar to promote the differentiation of these embryonic contacts into foreign motor synapses. In addition, the loss of electrical activity in the motoneuron, as opposed to the loss of postsynaptic potentials in the muscle fibers, appears to be responsible for these changes in connectivity. Our experiments suggest that neural activity may function during development by preventing inappropriate connections and thereby maintaining the precise connectivity achieved durIng nerve outgrowth and target selection.

[Key words: Drosophila, motoneurons, neural activity, neuromuscular junction, sprouting, synaptogenesis]

Neural activity plays a major role in establishing synaptic connections by influencing processes such as growth cone motility, target selection, and morphogenesis (reviewed by Goodman and Schatz, 1993). For example, in the vertebrate visual system, correlated synaptic activity between neighboring afferents is critical in refining the initially coarse connectivity. Synaptic activity directly regulates the segregation of visual inputs into alternating columns in mammals and experimentally manipulated lower vertebrates (Meyer, 1982; Schmidt and Edwards, 1983; Reh and Constantine-Paton, 1985; Stryker and Harris, 1986; Cline and Constantine-Paton, 1989).

Synaptic activity plays a similar role at the vertebrate neuromuscular junction (NMJ), where it regulates synapse elimination of the multiple motoneurons that initially innervate a muscle fiber. For example, chronic synaptic silencing increases the num-

\footnotetext{
Received June 14, 1995; revised Aug. 2, 1995; accepted Aug. 16, 1995.

We thank Drs. Hugo Bellen, Barry Ganetzky, and Corey Goodman for fly stocks and antibodies, and Marc Halfon, William Leiserson, Greg Lnenicka, and Robert Wyman for critically reading the manuscript. We thank all the members of the Keshishian lab for helpful discussions and advice. This work was supported by grants from the NIH and NSF to H.K.

Correspondence should be addressed to Jill Jarecki, Department of Biology, Yale University, P.O. Box 208103, New Haven, CT 06520-8103.

Copyright $\bigcirc 1995$ Society for Neuroscience 0270-6474/95/158177-14\$05.00/0
}

ber of multiply innervated muscle fibers, while prolonged neuromuscular stimulation leads to accelerated synapse elimination (Benoit and Changeux, 1978; O'Brien et al., 1978; Thompson, 1983). This occurs through competition, where active synaptic sites destabilize less active ones located on the same muscle fiber (Balice-Gordon and Lichtman, 1993). Synaptic activity can also regulate later events at the vertebrate NMJ. For example, blocking synaptic activity or denervating muscle fibers in adults results in the appearance of collateral sprouts derived from nearby nerve terminals (Brown and Ironton, 1977; Holland and Brown, 1980). These treatments cause the muscle fiber to revert to an earlier developmental state in which it reexpresses growth factors and adhesion molecules that promote motoneuron growth (Couvalt and Sanes, 1985; Reiger et al., 1985; Ishii, 1989; Caroni and Schneider, 1994).

Invertebrate systems, particularly Drosophila, are well suited for studying the role of synaptic activity during neuromuscular development. The Drosophila NMJ is a well-defined model system with uniquely identified pre- and postsynaptic cells that establish precisely specified connections (Keshishian et al., 1993). This allows for the observation and manipulation of single synapses throughout development. Moreover, several forms of plasticity occur at the Drosophila NMJ. The double mutant ether a go-go (eag) Shaker (Sh), which affects potassium channels and leads to enhanced nerve excitability, induces larval endings with increased numbers of branches and varicosities (Ganctzky and Wu, 1983; Budnik et al., 1990). Similar effects are seen with changes in cAMP-specific phosphodiesterase, CaM-dependent adenylyl cyclase, and CaM kinase I (Zhong et al., 1992; Wang et al., 1995). Moreover, embryonic denervation of muscles results in motor ending sprouting similar to that observed in vertebrates, demonstrating an additional example of plasticity (reviewed by Keshishian et al., 1993, 1994; Halfon et al., 1995).

Synaptic activity also influences early aspects of Drosophila NMJ development. For example, motoneuron excitation is required to localize glutamate receptors to the NMJ during embryogenesis (Broadie and Bate, 1993a,b). Embryonic synaptic transmission is first detected shortly after the growth cones contact their muscle targets (Broadie and Bate, 1993c). Therefore, synaptic activity may influence other aspects of synaptogenesis during this period, such as the refinement of connectivity. However, neither pathfinding nor target selection are affected by blocking activity (Broadie and Bate, 1993a). Furthermore, synapses remain polyinnervated throughout larval life (Keshishian et al., 1993), suggesting that synapse elimination, characteristic of vertebrate NMJs, does not occur in Drosophila.

Our experiments address the role of neural activity in maintaining the precise connectivity observed at the Drosophila NMJ during embryonic and larval development. Attention was focused on the induction of collateral sprouting, as this type of 
target plasticity results from muscle fiber denervation in Drosophila. In addition, in vertebrates the loss of synaptic activity is partially responsible for neuromuscular sprouting (Brown, 1984). Therefore, we reduced electrical activity during neuromuscular development both genetically and pharmacologically, using several mutants that affect $\mathrm{Na}$ channels as well as toxins that prevent synaptic activity. These experiments indicate that neural activity is involved in preventing innervation from secondary sources and that it may be involved in maintaining precise connectivity following its initial establishment.

\section{Materials and Methods}

Fly stocks

Flies were reared at room temperature $\left(22-25^{\circ} \mathrm{C}\right)$ unless otherwise indicated. Canton-S wild-type flics were used (CS-5, Woodard et al., 1989). The following mutants were used.

nap ${ }^{i s}$ : Adult flies and larvae paralyze at $37^{\circ} \mathrm{C}$ due to the failure of action potentials (Wu et al., 1978). The gene is allelic to male lethal and encodes a transcription factor that regulates expression of the para Na channel (Kernan et al., 1991; Kuroda et al., 1991).

para ${ }^{\prime s !}:$ Adults paralyze at $29^{\circ} \mathrm{C}$ and larvae at $37^{\circ} \mathrm{C}$ due to the failure of action potentials (Suzuki et al., 1971). These larva remain mostly physiologically normal at permissive temperatures (Siddiqi and Benzer, 1976; Wu and Ganetzky, 1980). The gene encodes one of the Na channels (Loughney et al., 1989).

para ${ }^{1 / 5}$ : It behaves as a null mutation with early first instar lethality (Ganetzky, 1984). It was maintained over a FM7c chromosome containing a $\beta$-galactosidase transgene, permitting the distinction of homozygous and hetcrozygous cmbryos using $\beta$-galactosidase labeling.

eag': It removes about $50 \%$ of $I_{\mathrm{K}}$ current. (Ganetzky and $\mathrm{Wu}, 1983$ ).

$S h^{120 b}$ : It reduces $I_{\mathrm{A}}$ current and prolongs EJPs (Ganetzky and Wu, 1983).

$s i^{t}$ : It is a recessive mutation with increased spontaneous activity in the adult giant fiber system although it induces paralysis at temperatures over $40^{\circ} \mathrm{C}$ (Kasbekar et al., 1987). Binding studies indicate that the number of $\mathrm{Na}$ channels are reduced at all temperatures (Jackson et al., 1984, 1985). Na current density is reduced in embryonic neurons (O'Dowd and Aldrich, 1988). The gene encodes an eag family potassium channel (L. Hall, personal communication). At this time the mechanism of paralysis remains unclear for this mutation.

$s e i^{2}$ : This is a semidominant mutation with increased spontaneous activity in the adult giant fiber system (Kasbekar et al., 1987). It changes the affinity of ligand binding to Na channcls, but not channel numbers or Na current density (Jackson et al., 1984, 1985; O'Dowd and Aldrich, 1988). At this time the mechanism of paralysis remains unclear for this mutation.

tipE: Adults paralysis at $40^{\circ} \mathrm{C}$ due to the failure of action potentials (Kalkarni and Padhye, 1982). It encodes a novel transmembrane protein, which may be an auxiliary protein essential for para $\mathrm{Na}$ channel function (Feng et al., 1995).

$y$ (l) $l^{d 2.3} f^{36 a} \mathrm{car}$ : This is a lethal deletion that lies close to para but does not uncover it (Stern et al., 1990).

$D p(1 ; 4) r^{+} f^{+}$: This duplication carries the region $14 \mathrm{~A}-16 \mathrm{~A} 2$ attached to the fourth chromosome. It covers the para gene as well as (l) $l^{d 2.3}$. Its presence was scored by $f^{+}$(Stern et al., 1990). This stock and the preceding one were used for generating para duplications.

$s y t^{A D I}$ and $s y t^{T A l}$ : These alleles have first instar lethality and were crossed to generate transheterozygotes that survive until eclosion. In $\mathrm{AD} 1 / \mathrm{T} 41$ third instar larvae evoked PSPs show a $75 \%$ reduction (Littleton et al., 1994).

\section{Temperature shifts}

Embryos. Mutant embryos were collected for $2 \mathrm{hr}$ at room temperature. For some experiments they were reared continuously at $18^{\circ} \mathrm{C}$ until dissection. In others, they were raised at $18^{\circ} \mathrm{C}$ until stage 15 , at which time they were shifted to $34^{\circ} \mathrm{C}$ for $2.5 \mathrm{hr}$ until late stage $16 /$ early stage 17. At this stage, all embryos were dissected, fixed, and labeled with antibodies to fasciclin II (Van Vactor et al., 1993). In the case of the paralk. embryos, they were also stained with anti $\beta$-galactosidase (Promega).

Third instar larvae. Third instar larvae reared at room temperature were collected from vials containing adult flies. In order to obtain temperature-shifted third instar larvae, embryos were collected at room temperature for $2 \mathrm{hr}$, and then were allowed to develop at $18^{\circ} \mathrm{C}$ until early stage 15 . Then they were temperature-shifted to $34^{\circ} \mathrm{C}$ for $6 \mathrm{hr}$ until the end of embryogenesis, at which point they were down-shifted to $25^{\circ} \mathrm{C}$ for $18 \mathrm{hr}$. During subsequent larval development they were temperature shifted from $25^{\circ} \mathrm{C}$ to $37^{\circ} \mathrm{C} 6 \mathrm{hr} / \mathrm{d}$. At the wandering third instar stage, larvae were dissected, fixed, and stained with anti-HRP.

\section{Dissection and staining}

Dissection and immunocytochemistry of both embryos and larvae were done as described by Halpern et al. (1991). Third instar larvae were often heat-fixed in $60^{\circ} \mathrm{C}$ water for $5 \mathrm{sec}$ prior to dissection. Antibodies to fasciclin Ir (Van Vactor et al., 1993), fasciclin III (Patel et al., 1987; Snow et al., 1989), mouse anti $\beta$-galactosidase (Promega), and goat anti-HRP (Cappel) were used. Primary antibodies were visualized using peroxidase-linked secondary antibodies (Vectastain elite ABC kit, Vector Labs; donkey anti-goat, Cappel). X-gal staining for $\beta$-galactosidase detection was performed as described by Bellen et al. (1989) overnight at $37^{\circ} \mathrm{C}$.

\section{Filleted embryo culture}

Embryos dissected at early stage 16 (13 hr AEL) as determined by gut development were cultured in L15 medium without glutamate (Sigma). FCS (5\%; Hyclone), $10 \mathrm{mg} / \mathrm{ml}$ glucose, $2 \mathrm{mg} / \mathrm{ml}$ trehalose, $0.125 \mathrm{U} / \mathrm{ml}$ insulin (Sigma), $50 \mathrm{U} / \mathrm{ml}$ penstrep (Sigma), and $1 \mathrm{ng} / \mathrm{ml} \beta$-ectosterone (Sigma) were added to the medium. Fmbryos were filleted in saline, which was replaced with $\mathrm{L} 15$ after dissection. In order to allow oxygen exchange, embryos were barely submerged with L15. Neural activity was blocked by the addition of TTX, at a final concentration of $10 \mu \mathrm{M}$. Glutamate receptor activation was blocked by the addition of AR636 at a final concentration of $0.01 \mu \mathrm{g} / \mu \mathrm{l}$. Embryos developed for $4-5 \mathrm{hr}$ at $25^{\circ} \mathrm{C}$ in a humid chamber until early stage 17 . Until this point NMJ development proceeded normally. However, prolonged culturing during stage 17 led to the deterioration of the muscles although the cuticle, mouth hooks, and denticle belts continued to develop. The embryos were fixed and stained as described above. A similar culture system was developed by Broadie et al. (1992).

\section{Toxin Injection}

Toxin injections were performed as described by Keshishian et al. (1993). The toxins were injected into intact animals at early stage 16 (13 hr AEL). The embryos were allowed to develop for $4 \mathrm{hr}$ at $25^{\circ} \mathrm{C}$. They then were dissected, fixed, and stained with anti-HRP (Johansen et al., 1989b).

\section{Results}

Our experiments indicate that decreasing neural activity promotes the appearance of ectopic neuromuscular synapses in Drosophila. This was shown using mutants that affect Na channels, such as paralytic (para), no action potential (nap), temperatureinduced paralysis $E$ (tipE), and seizure (sei) (for review see Ganetzky and Wu, 1986) and pharmacological agents such as TTX, which prevents action potentials, and the orb-web spider toxin AR636, which abolishes glutamate receptor activation (reviewed by Jackson and Underwood, 1988). We observed collateral sprouts in the embryos, which correlated with the presence of ectopic synapses in the third instar larvae. Furthermore, neural activity must be reduced during both the embryo and the first instar to promote the differentiation of these filopodial-like sprouts into the ectopic synapses seen in third instar larvae. Our results indicate that neural activity is not required to form the general pattern of connectivity but prevents innervation from inappropriate secondary sources later in development.

\section{The Drosophila $N M J$}

Because the Drosophila NMJ is well characterized, the effect of synaptic activity blockade could easily be assessed. There are 30 uniquely identifiable muscle fibers per abdominal hemisegment (A2-A7), which are innervated by about 35 motoneurons 


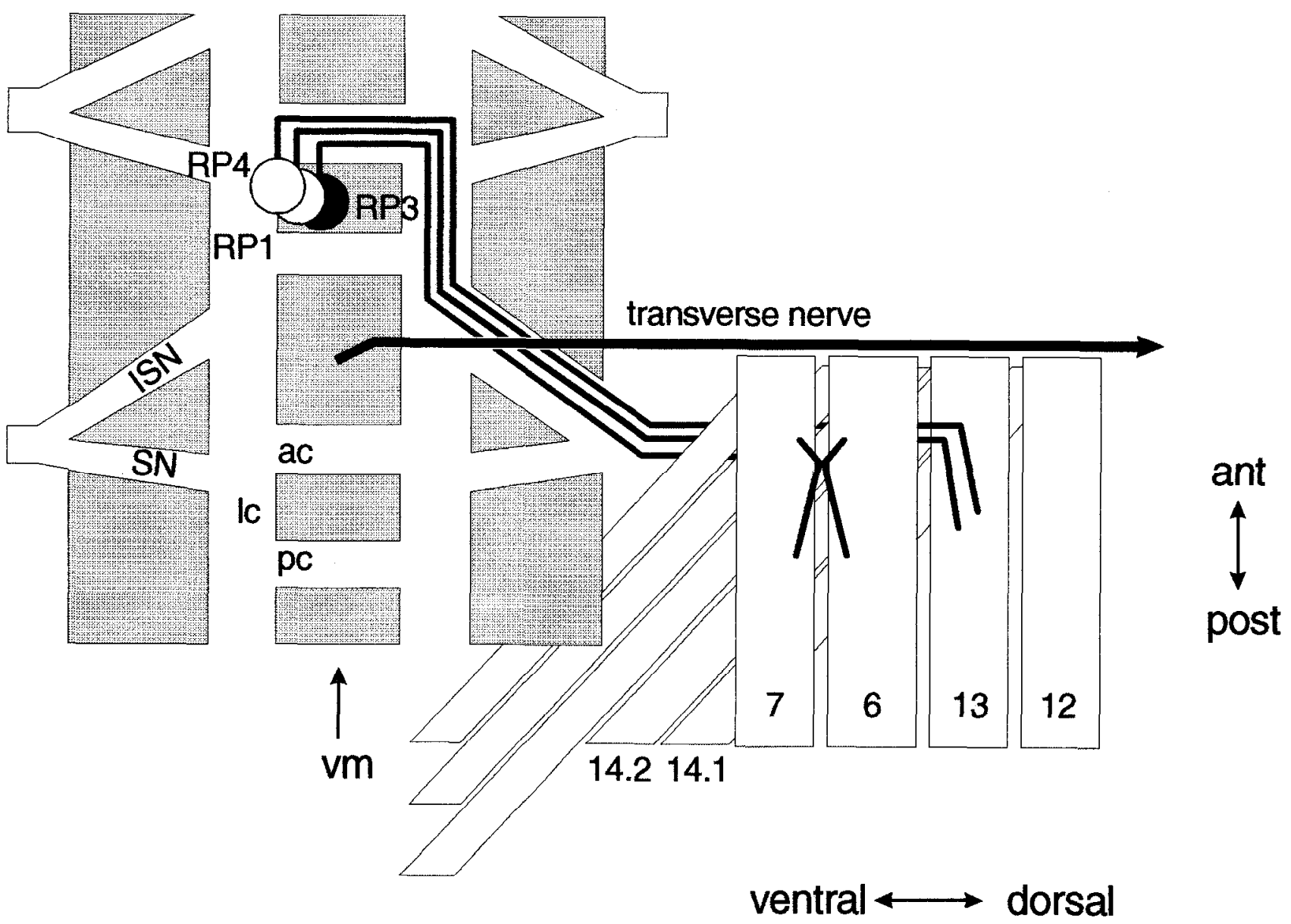

Figure 1. A schematic representation of the motoneuron connectivity in one abdominal hemisegment of an embryonic Drosophila bodywall. Anterior is up and ventral is to the left. The ventral longitudinal muscle fibers 7, 6, 13, and 12 and the identified motoneurons that innervate them are shown. The CNS is to the left, shaded in gray. The anterior and posterior commissures, the longitudinal connectives, and the two main nerve tracts (SN and ISN) are shown in outline. Motoneuron RP3, which innervates muscle fibers 6 and 7 in their shared cleft, is black. Motoneurons RP1 and RP4, which innervate muscle fiber 13, are white. The transverse nerve (TN), which runs along the segmental border of the ventral longitudinal muscles but normally does not innervate them, is shown in black.

located in the ventral nerve cord (shown schematically in Figure 1; Johansen et al., 1989a; Bate, 1990; Sink and Whitington, 1991a). Each motoneuron innervates a specific muscle target, resulting in precise connectivity at all muscle fibers. For example, the motoneuron RP3 always innervates muscle fibers 6 and 7 at their shared cleft while RP1 invariably innervates the adjacent muscle fiber 13 (Fig. 1; Halpern et al., 1991; Sink and Whitington, 1991a,b). During mid-embryogenesis motoneuron axons exit the CNS in each hemisegment within the two main nerves, the segmental and intersegmental nerves (SN and ISN). The growth cones first contact their specific muscle targets several hours later during early stage 16 (Johansen et al., 1989b; Broadie and Bate, 1993c). Synaptic transmission begins shortly after the growth cones contact their muscle targets (Broadie and Bate, 1993c). The original contacts are immunopositive for glutamate, which is the main neurotransmitter at the Drosophila NMJ (Jan and Jan, 1976a,b; Johansen et al., 1989b), and are capable of evoking PSPs (Broadie and Bate, 1993c). Even though the embryonic motor ending is active and prefigures the mature larval ending in both synapse location and shape, it exhibits an immature morphology (Johansen et al., 1989a). It is not until the last hours of embryogenesis that the synapse differentiates its first synaptic boutons. During the first instar stage, the different ending types (type I with large boutons and type II with small boutons) become distinguishable. During larval life, the NMJ continues to develop, with large increases in bouton and branch number as well as muscle fiber surface area (Keshishian et al., 1993). At this point, the highly stereotypic ending morphology of each muscle fiber can be observed (Johansen et al., 1989b).

\section{Activity mutants that affect Na channels display ectopic neuromuscular synapses in third instar larvae.}

To determine the role of neural activity in maintaining correct synaptic connectivity, activity mutants that affect $\mathrm{Na}$ channels, such as paralytic, no action potential, temperature induced paralysis $E$, and seizure were analyzed during the third larval instar for the presence of ectopic neuromuscular synapses similar to those induced after denervation. These mutations directly affect action potentials in the neuron since $\mathrm{Na}$ conductances do not exist in the muscle fibers. Two different classes of mutations were examined in this study. The first consists of mutations in $\mathrm{Na}$ channel proteins. There are two characterized Drosophila $\mathrm{Na}$ channels, but only one, paralytic, has mutations (Salkoff et al., 1987; Loughney et al., 1989). paralytic is expressed ubiquitously in embryonic and larval neurons (Hong and Ganetzky, 1994). 

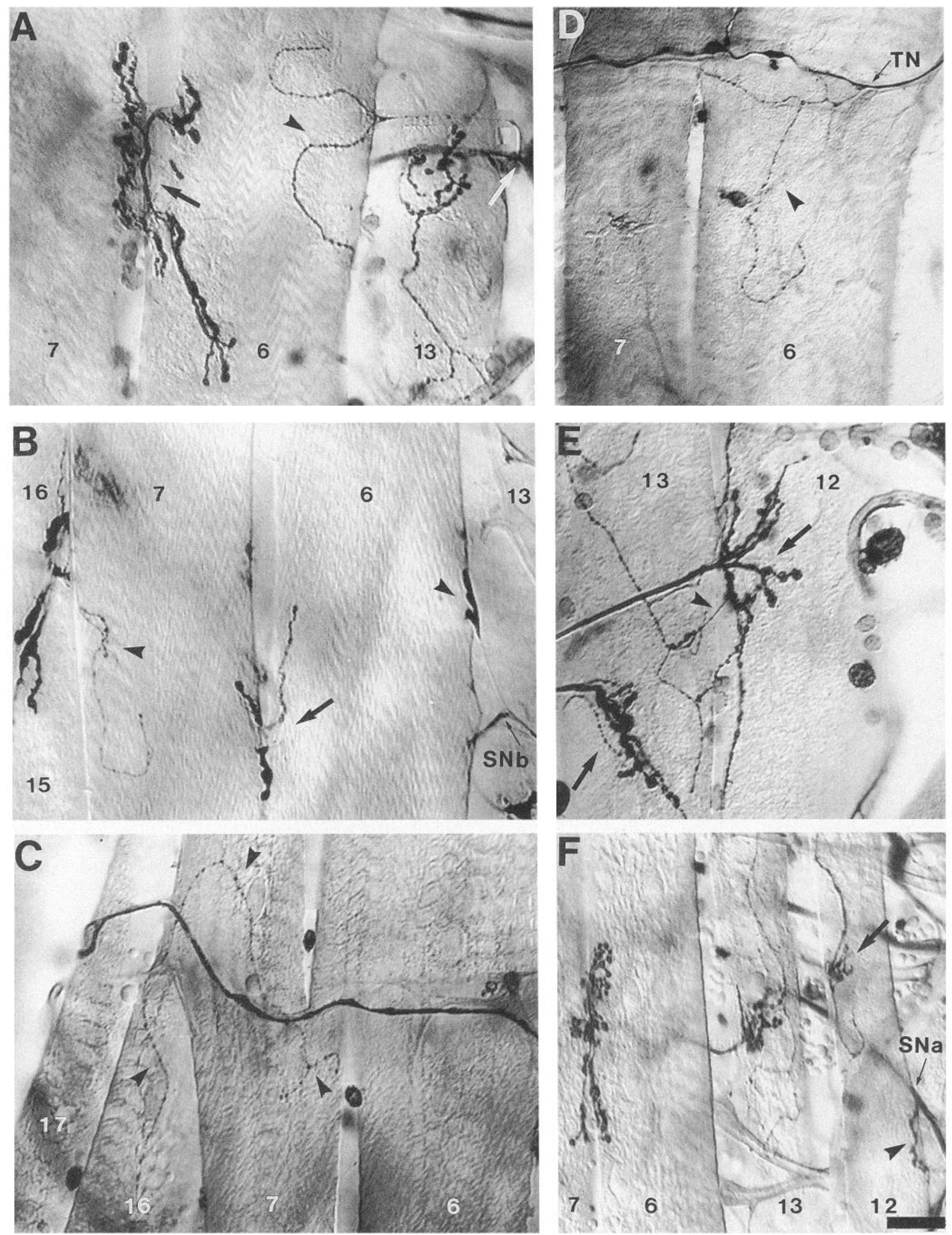

Figure 2. Ectopic motoneuron endings were observed on muscle fibers throughout the bodywall of activity mutant third instar larvae. Anterior is up and ventral is to the left. Neurons are labeled with antibodies to horseradish peroxidase. $A$, An ectopic ending extends back from the SNb branch 
para $^{\text {ts }}$, a hypomorphic mutation in the para Na channel protein, was used in this study as it does not affect larval viability (Siddiqi and Benzer, 1976). This mutant paralyzes at elevated temperatures in both the embryo and the adult, but does not show a reduction in the number of $\mathrm{Na}$ channels in ligand binding studies at any temperature (Kauvar, 1982). Moreover, it does not have many phenotypes at the permissive temperature, such as increased I"IX sensitivity or changes in refractory period (Siddiqi and Benzer, 1976; Wu and Ganetzky, 1980).

The sccond class of mutations, which affects Na channel expression or function, was also analyzed. This class includes $n a p^{t s}$, tipE, and $s e i^{l}$. nap encodes a transcription factor that regulates para $\mathrm{Na}$ channel expression (Kernan et al., 1991; Kuroda et al., 1991), tipE encodes a novel transmembrane protein that may be an auxiliary protein required for $\mathrm{Na}$ channel function (Feng et al., 1995), and seizure encodes a eag family potassium channel (Hatl, personal communication). All three mutants show reductions in ligand binding to $\mathrm{Na}$ channels in adult $\mathrm{CNS}$ extracts at high and low temperatures (Kauvar, 1982; Jackson et al., 1984, 1985, 1986). Moreover, in $s e i^{\prime}$ and tipE embryonic neurons, $\mathrm{Na}$ current densities are reduced while in $\mathrm{para}^{\mathrm{ts}}$, there are $25 \%$ fewer neurons with $\mathrm{Na}$ conductances (O'Dowd and Aldrich, 1988; O'Dowd et al., 1989). In contrast, Na current densities were unaffected in napts embryonic cultured neurons, although nap protein is expressed during late embryogenesis (O'Dowd et al., 1989; Kuroda et al., 1991).

We analyzed mutant third instar larvae after they had developed in either of two conditions. Animals were raised at room temperature throughout their entire embryonic and larval development or were temperature shifted to $37^{\circ} \mathrm{C}$ for $6 \mathrm{hr} / \mathrm{d}$ from late embryogenesis through the third instar. For these mutants, $37^{\circ} \mathrm{C}$ is near the restrictive temperature, but prolonged exposure to this temperature is lethal even to wild-type animals (see Ashburner, 1989). Therefore, temperature shifting $6 \mathrm{hr} / \mathrm{d}$ allowed for substantial activity block during that period, while still ensuring the survival of the larvae.

Neuromuscular connectivity was first assessed by analyzing the innervation on the ventral longitudinal muscle fibers 6 and 7 in third instar larvae. An increase in the frequency of ectopic endings on these muscle fibers was observed in $n a p^{i s}, t i p E$, and $s e i^{\prime}$ larvae raised at all temperatures. Ectopic endings refer to motoneuron terminals on a muscle fiber that are located at un usual sites and arise from inappropriate sources. Therefore, innervation from inappropriate motoneurons located at the site of native innervation would remain undetected. The ectopic endings are morphologically similar to those induced by muscle fiber denervation, and arise from the same nerve sources (Fig. 2; Keshishian et al., 1993, 1994; Halfon et al., 1995). Ectopic endings in third instar larvae originate from three nerve sources. Five percent come from $\mathrm{SNb}$ motoneurons that innervate muscle fibers 13 and 12 (Fig. $2 A, B$ ). Another $25 \%$ come from SNd branches that innervate the ventral oblique muscle fibers 15 and 16 (Fig. $2 B, C$ ). The majority of ectopic endings, $70 \%$, originate

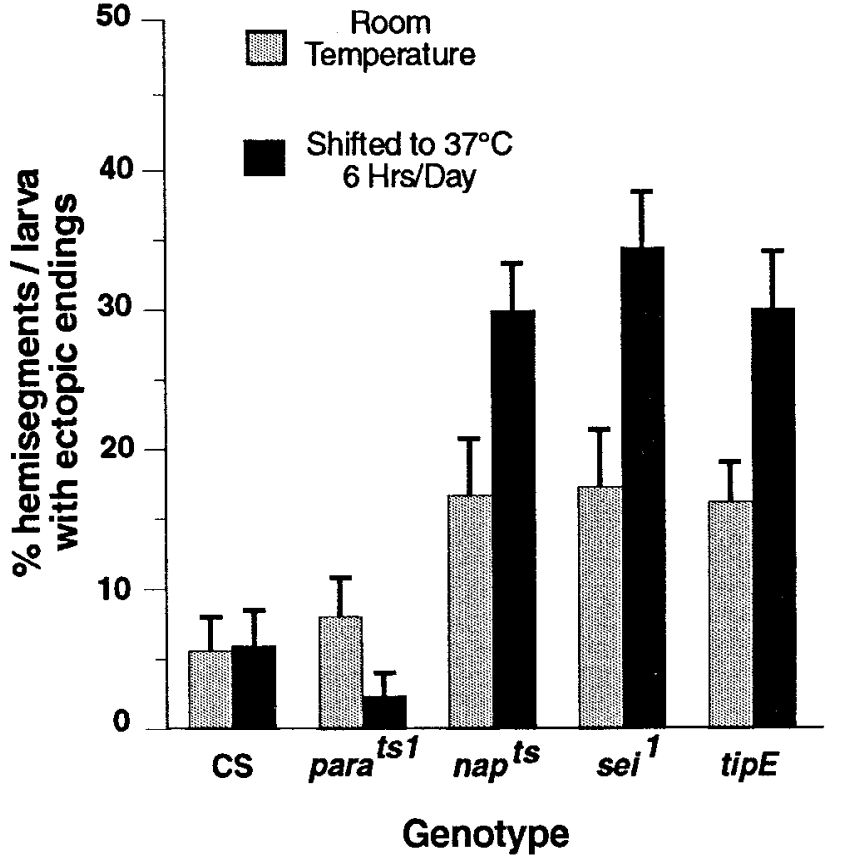

Figure 3. The frequency of ectopic endings in mutant third instar larvae. In $n a p^{t s}, s e i^{\prime}$, and tipE, about a threefold increase in the frequency of ectopic endings is observed in larvae raised at room temperature, compared to $\mathrm{CS}$ wild type ( $p<0.025$ by $t$ test). Atter rearing the animals at $37^{\circ} \mathrm{C}$ for $6 \mathrm{hr} / \mathrm{d}$, there is an additional twofold increase. The temperature-induced increase was significant in the mutants when compared to CS temperature-shifted animals $(p<0.005)$ and to their own room temperature levels $(p<0.01$ ). However, the frequency of ectopic endings in paratst larvae was not different from wild type at either temperature. $n=\mathrm{CS}$ : room temperature (RT), 89 heisegments in 8 animals; CS temperature-shifted (TS), 68 in 9 . nap ${ }^{i s}$ RT, 76 in 15 ; nap is $^{i s}$ TS, 86 in 12; tipE RT, 116 in 20; tipE TS, 96 in 12; para ${ }^{s / s}$ RT, 116 in 17; para ${ }^{\text {st }} \mathrm{TS}, 89$ in $10 \mathrm{sei}^{I} \mathrm{RT}, 109$ in 21 ; sei $i^{\prime} \mathrm{TS}, 129$ in 13.

from the transverse nerve, which normally runs along the segmental boundary of these muscle fibers but rarely innervates them (Fig. 2D). This nerve carries sensory input as well as efferent axons that innervate the allary muscle fiber and muscle fiber 25 (Gorczyca et al., 1994). Most ectopic endings originated from axons as opposed to nerve terminals. They showed typical motor ending anatomy, including the several classes of boutons previously described (Atwood et al., 1993).

A threefold increase over the wild-type frequency of ectopic endings on muscle fibers 6 and 7 was observed $n a p^{t s}$, $s e i^{l}$, and tipE larvae raised at room temperature. The frequency increased from $5.8 \%$ of hemisegments/larvae in Canton-S (CS) to almost $17 \%$ in each of the three mutants ( $p<0.025$; Fig. 3 ). An additional twofold increase to at least $30 \%$ was observed after the temperature shift regimen. This temperature-induced increase was significant in $n a p^{t s}$, sei $i^{l}$, and tipE when compared both to CS temperature-shifted animals $(p<0.005)$ and to their own room temperature levels ( $p<0.01$; Fig. 3 ). The hypomorphic

innervating muscle fiber 12 (white arrow) onto muscle fiber 6 (black arrowhead). The native ending on muscle fiber 6 is unaffected (black arrow). $B$, An ectopic ending on muscle fiber 7 (black arrowhead) originates from the SNd nerve branch innervating muscle fibers 16 and 15 . An ectopic ending from the $S \mathrm{Nb}$ branch extends back onto muscle fiber 6 (black arrowhend). The mative ending on muscle fiber 6 and 7 is unaffected (black arrow). $C$, An ectopic ending originating from the ending on muscle fiber 16 innervates muscle fiber 7 in two adjacent segments (black arrowheads). $D$, An ectopic ending originating from the transverse nerve innervates muscle fiber 6 (black arrowhead). E, An ectopic ending originating from the native ending on muscle fiber 12 (black arrow) innervates muscle fiber 13 (black arrowhead). The native ending on muscle fiber 13 is shown (black arrow). F, Ectopic ending on muscle fiber 12 (black arrowhead) originates from the SNa branch that innervates the muscle fibers just dorsal to muscle fiber 12. The native ending on muscle fiber 12 is unaffected (black arrow). Scale bar: $25 \mu \mathrm{m}$ for $A-E, 40 \mu \mathrm{m}$ for $F$. 
Table 1. Ectopic endings on muscle fibers 6 and 7 in third instar larvae

\begin{tabular}{|c|c|c|}
\hline \multirow[b]{2}{*}{ Genotype } & \multicolumn{2}{|c|}{$\begin{array}{l}\% \text { Hemisegments/larvae with an } \\
\text { ectopic ending }{ }^{a}\end{array}$} \\
\hline & $\begin{array}{l}\text { Reared at room } \\
\text { temperature }\end{array}$ & $\begin{array}{l}\text { Reared at } 37^{\circ} \mathrm{C} \\
6 \mathrm{hr} / \mathrm{d}\end{array}$ \\
\hline$C S$ & $5.8 \pm 2(8,89)$ & $6 \pm 3(9,68)$ \\
\hline$n a p^{t s} ; D p(1 ; 4) r^{+} f^{+}$ & $9.6 \pm 2(19,174)$ & $9.6 \pm 1(29,250)$ \\
\hline eag sh & $0 \pm 0(5,39)$ & $8 \pm 2(13,99)$ \\
\hline$s e i^{2}$ & $8 \pm 4(9,80)$ & $11 \pm 4(13,120)$ \\
\hline naps; tipE & $24 \pm 2(34,298)^{* \dagger}$ & $40 \pm 3(17,141) \neq$ \\
\hline para $^{\text {sII }} ;$ tipE & $22 \pm 4(21,188)^{*}$ & $40 \pm 2(19,162) \ddagger$ \\
\hline
\end{tabular}

${ }^{a}$ Average \pm SEM, $n$ (animals, hemisegments).

* Significantly greater than CS room temperature $(p<0.005$ by Student's $t$ test).

$\dagger$ Significantly greater than single allele at room temperature $(p<0.05$ by Student's $t$ test).

Significantly greater than double mutant at room temperature $(p=0.025$ by Student's $t$ test) and single mutant after temperature shift $(p<0.005)$.

para $^{\text {st }}$ mutant, however, did not increase the levels of ectopic endings at any temperature, perhaps because this mutant does not have phenotypes at the permissive temperature (Siddiqi and Benzer, 1976; Wu and Ganetzky, 1980; Kauvar, 1982).

Our data suggest that the increase in the number of ectopic endings in the activity mutants is not due to background influences, but specifically to reductions in neural activity. $n a p^{t s}, s e^{\prime}$, and tipE all show a temperature-sensitive increase in the frequency of ectopic endings unlike CS (Fig. 3) or Oregon R (data not shown) wild-type larvae. Moreover, a duplication of the paralytic gene, which previously has been shown to specifically rescue temperature-induced paralysis in napts animals (Stern et al., 1990), prevents the appearance of ectopic endings in nap ${ }^{t s}$ larvae in our study (Table 1). Therefore, it is unlikely that background influences or that other genes whose expression could be regulated by this transcription factor cause ectopic ending to appear in napts larvae. It is much more likely that this phenotype is due to loss of $\mathrm{Na}$ current in the motoneuron.

Several mutants that increasc ncural activity were also analyzed in order to determine if ectopic ending formation occurs specifically in response to decreased activity or alternatively to deviations in either direction. In the eag sh double mutant, which affects potassium channels and leads to nerve hyperexcitability, the number of ectopic endings on muscle fibers 6 and 7 did not increase at either temperature compared to wild type (Table 1; Ganetzky and Wu, 1983). A second allele of seizure, sei ${ }^{2}$, was also analyzed. seizure encodes a potassium channel, and both alleles have a hyperactive phenotype in the adult giant fiber system (Kasbekar et al., 1987; L. Hall, personal communication). However, sei $i^{\prime}$, which has both a lower number of $\mathrm{Na}$ channels and a lower $\mathrm{Na}$ current density, increases the frequency of ectopic endings at any temperature, while $s e i^{2}$, which shows neither of these phenotypes, does not (Table 1; Jackson et al., 1984, 1985, 1986; O'Dowd and Aldrich, 1988). Thus, the appearance of ectopic endings is specifically dependent on reductions in neural activity.

\section{Double mutants further increase the frequency of ectopic endings}

Neural activity is not totally blocked in any of the single mutants examined. Two double mutants were generated to determine
Table 2. Ectopic endings on muscle fiber 12 in temperature shifted third instar larvae

\begin{tabular}{ll} 
Genotype & $\begin{array}{l}\text { \% Hemisegments/larvae with an } \\
\text { ectopic ending }\end{array}$ \\
\hline$C S$ & $7.3 \pm 2(9,71)$ \\
$n a p^{t s}$ & $25 \pm 4(11,83)^{*}$ \\
tipE & $16 \pm 3(12,95)^{*}$ \\
sei $i^{l}$ & $19 \pm 5(11,86)^{*}$
\end{tabular}

${ }^{a}$ Average \pm SEM, $n$ (animals, hemisegments).

* Significantly greater than CS ( $p<0.025$ by Student's $t$ test)

whether the frequency of ectopic endings is increased in more severe mutant combinations. Both nap ${ }^{t s}$; tipE and para $^{\text {tsl}}$; tipE adults have a greater reduction in $\mathrm{Na}$ channel number than, paralyze at much lower temperatures than, and show reduced viability in comparison to either corresponding single mutant (Ganetzky, 1984, 1986; Jackson et al., 1986). At room temperature the double mutants have a slight increase in the number of ectopic endings in third instar larvae. nap $p^{t s}$;ipE third instar larvae have a frequency of ectopic endings of $24 \%$ of hemisegments/ animals, and the frequency in para $^{\text {tsl }}$; tipE larvae is $22 \%$ (Table $1)$. This increases to $40 \%$ in both double mutants after undergoing temperature shift $(p<0.005$ when compared to double mutant reared at room temperature and $p=0.025$ when compared to temperature-shifted single mutants; Table 1). The frequency of ectopic ending correlates with the extent of the activity block. Thus, the further that neural activity is reduced the greater the number of ectopic endings that will appear.

\section{Ectopic endings are observed on muscles throughout the body wall}

The mutants are expected to affect neural activity in all motoneurons. Therefore, increases in the frequency of ectopic endings should be seen on muscle fibers throughout the body wall. As predicted, we observed them on many different muscle fibers, not just on muscle fibers 6 and 7. For example, in Figure $2 E$, the native motor ending of muscle fiber 12 extends a process back onto muscle fiber 13. In this case the ectopic ending comes from the same nerve branch that normally innervates muscle fiber 13. In Figure $2 F$, muscle fiber 12 receives an ectopic ending from $\mathrm{SNa}$, a nerve that normally innervates mid-bodywall muscle fibers dorsal to 12 . Throughout the body wall, the native endings appear to be unaffected by the ectopic synapses. The number of ectopic endings on muscle fiber 12 was quantified for CS, and for the $n a p^{t s}$, $s e i^{l}$, and tipE mutants, which had undergone the temperature shift regimen. A significant increase in the number of ectopic endings was observed on muscle fiber 12 in all of the mutants compared to CS control larvae (Table 2). In addition, cctopic endings were observed on the dorsal body wall muscles. For example, several ectopic endings, orginating from the transverse nerve were observed on muscle fiber 5. Multiple example of ectopic endings, arising from SNa nerve branch that innervates the transverse muscle fibers, were seen on muscle fiber 4 (data not shown). The fact that ectopic endings are observed throughout the entire body wall supports the hypothesis that ectopic ending formation is promoted by hypoactivity. 

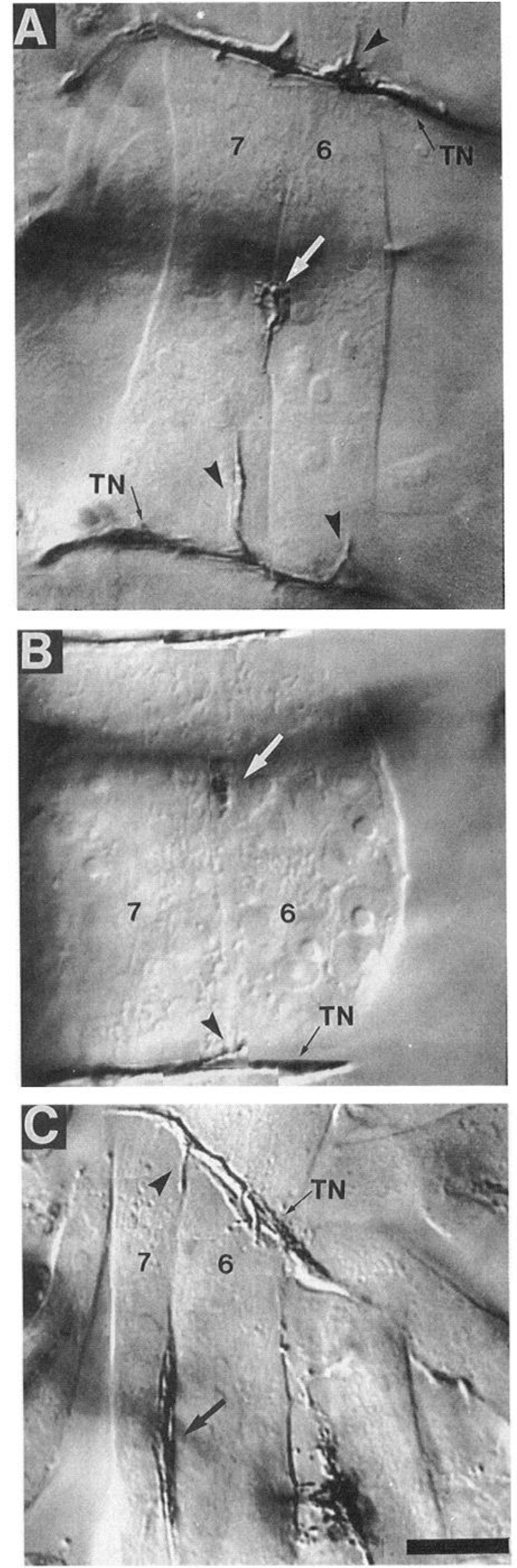

\section{Collateral sprouting is first observed during late embryogenesis}

To determine the time course for the formation of ectopic endings, mutant embryos were analyzed for inappropriate innervation. It was now possible to use para ${ }^{\text {ths }}$, which behaves as a null mutant with first instar lethality (Ganetzky, 1984), as well as the other mutant lines previously described. The para embryos have previously described electrophysiological and behavioral phenotypes. For example, synaptic transmission fails in these embryos at $34^{\circ} \mathrm{C}$ (Broadie and Bate, 1993a). Therefore, embryos were reared at either $18^{\circ} \mathrm{C}$ or $34^{\circ} \mathrm{C}$. Rearing para embryos at $34^{\circ} \mathrm{C}$ promotes the appearance of immature filopodial contacts on muscle fibers 6 and 7. We refer to these as collateral sprouts. Every collateral sprout originates from the transverse nerve (Fig. $4 A$ ), unlike the third instars, where ectopic endings arise from several nerve sources. The frequency of collateral sprouting on muscle fibers 6 and 7 increases in the para $a^{\mid s t}$ and para $^{l k 5}$ mutants from about $10 \%$ of hemisegments in wild-type animals to $30 \%$ in para animals reared at the restrictive temperature $(p<0.005$; Fig. $5 A$ ). In addition, para ${ }^{k s}$ heterozygotic embryos also show a similar temperature sensitive increase in collateral sprouting on muscle fibers 6 and 7 from $17 \%$ of hemisegments at $18^{\circ} \mathrm{C}$ to $27 \%$ at $34^{\circ} \mathrm{C}\left(p<0.005 ; n: 18^{\circ} \mathrm{C}, 106\right.$ hemisegments in 12 embryos; $34^{\circ} \mathrm{C}, 116$ heisegments in 13 embryos). These increases are not due to the high temperature itself, but to the resulting failure in action potentials. For example, para ${ }^{t s /}$ embryos reared at $32^{\circ} \mathrm{C}$, a temperature at which the para $a^{t s l}$ embryos remain active as assessed both behaviorally and electrophysiologically (Broadie and Bate, 1993a), did not show an increase in sprouting ( $12 \%, n=156$ segments in 15 animals). Therefore, collateral sprouting begins during embryogenesis and correlates with the failure of action potentials, not with adverse affects of elevated temperature.

There is also evidence that the nap, tipE, and sei protein products function during embryogenesis. For example, tipE and $s e i^{\prime}$ have reduced $\mathrm{Na}$ current density in the embryo, and nap is is at least expressed during this period (O'Dowd et al., 1989; Kuroda et al., 1991). The nap ${ }^{t s}$, tipE, and $s e i^{l}$ mutants show increases in the levels of collateral sprouting on muscle fibers 6 and 7 that are comparable to temperature-shifted para embryos. However, this increase is observed in animals reared at both $18^{\circ} \mathrm{C}$ and $34^{\circ} \mathrm{C}$ (Fig. $5 \mathrm{~B}$ ). The embryonic restrictive temperatures for these mutants is unknown. However, unlike para $a^{t s l}$, these mutants show a number of permissive temperature phenotypes, so it is not unexpected that permissive-temperature sprouting occurs (Siddiqi and Benzer, 1976; Wu and Ganetzky, 1980; Ganetzky and $\mathrm{Wu}, 1986$ ).

\section{The critical period for the induction of ectopic endings includes both late embryogenesis and the first instar}

$n a p^{t}$, $s e i^{i}$, and tipE animals were temperature shifted during different periods of development in order to identify times when

\section{$\leftarrow$}

Figure 4. Reducing neural activity in embryos by either pharmacological or genetic means increases the frequency of collateral sprouts from the transverse nerve onto muscle fibers 6 and 7 . The muscles remain normally innervated in their shared cleft (large arrows), but collateral sprouts (arrowheads) appear on the muscle fibers arising from the transverse nerve $(T N)$. A, Late stage 16/early stage 17 tipE embryo reared at $18^{\circ} \mathrm{C} . B$, Late stage 16 cultured embryo treated with TTX. $C$, A late stage $16 /$ early stage 17 embryo injected with TTX. $A$ and $B$, labeled with antibodies to horseradish peroxidase; $C$, with anti-fasciclin II. Anterior is up and ventral to the left. Scale bar, $10 \mu \mathrm{m}$. 


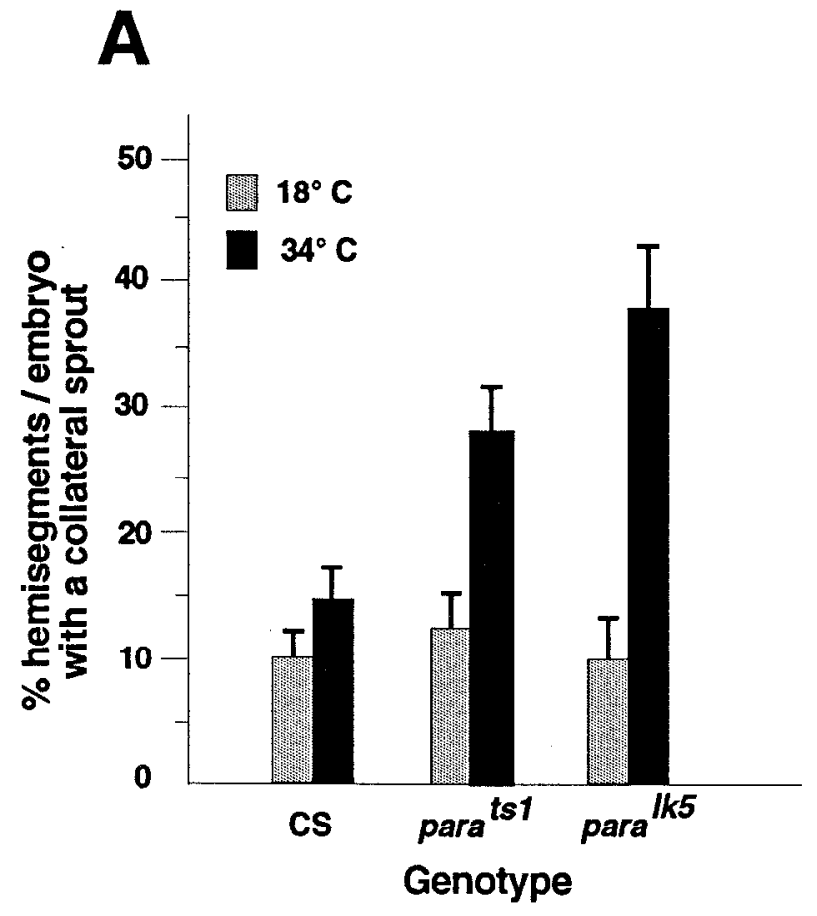

B

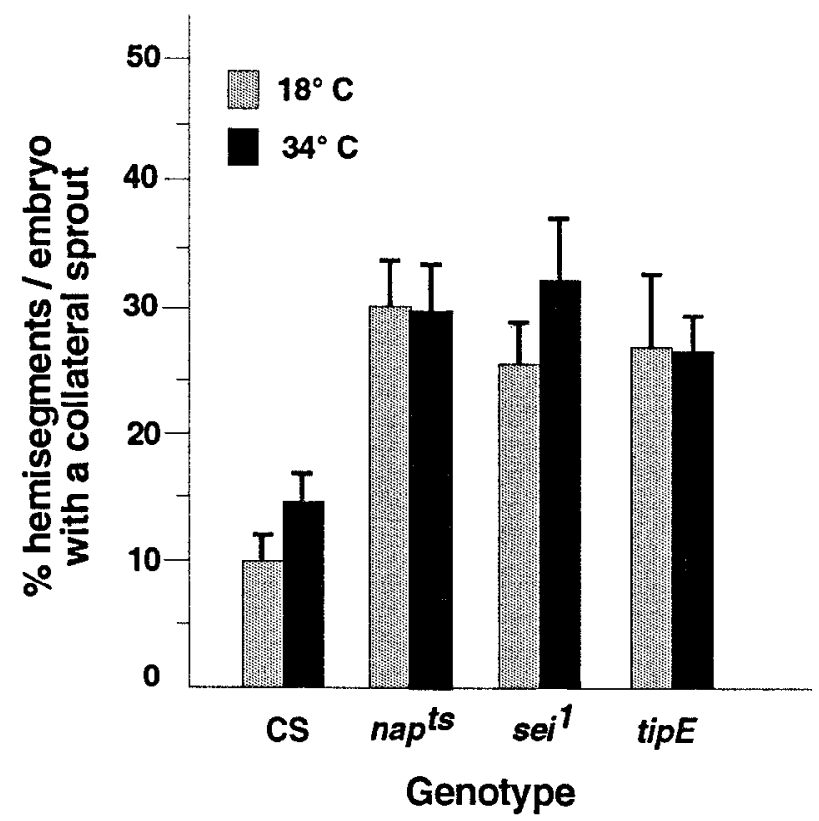

Figure 5. The frequency of collateral sprouting increases in embryos with mutations that affect $\mathrm{Na}$ channels. A, Two alleles of the paralytic gene that encodes a $\mathrm{Na}$ channel, para $^{t s /}$ and para ${ }^{k .5}$, show increases in collateral sprouting only at the restrictive temperatures, at which they are paralyzed ( $p<0.005$ by $t$ test). $n$ : CS $18^{\circ} \mathrm{C}, 111$ hemisegments in 13 animals; $\operatorname{CS~} 34^{\circ} \mathrm{C}, 214$ in 24 ; para ${ }^{\text {ss }} 18^{\circ} \mathrm{C}, 143$ in 15 ; para ${ }^{i s l} 34^{\circ} \mathrm{C}$, 200 in 22 ; para $^{l k 5} 18^{\circ} \mathrm{C}, 64$ in 7 ; para ${ }^{l k 5} 34^{\circ} \mathrm{C}, 82$ in 9 . B. Mutants of $n a p^{t s}, s e i^{\prime}$, and tipE, which do not encode $\mathrm{Na}$ channels but do influence the number of functional $\mathrm{Na}$ channels at all temperatures in binding studies, show similar increases in the frequency of collateral sprouting at both $18^{\circ} \mathrm{C}$ and $34^{\circ} \mathrm{C}$ ( $p<0.005$ by $t$ test). $n$ : nap $p^{t s} 18^{\circ} \mathrm{C}, 255$ in 27 ; nap ${ }^{t s} 34^{\circ} \mathrm{C}, 138$ in 19 ; sei $18^{\circ} \mathrm{C}, 178$ in 19 ; sei $34^{\circ} \mathrm{C}, 160$ in 19 ; tip $^{\prime}$ $18^{\circ} \mathrm{C}, 157$ in 15 ; tip $34^{\circ} \mathrm{C}, 159$ in 15 . the reduction in activity is required for the enhanced sprouting phenotype observed following the temperature shift regimen (Fig. 6). When these mutants were reared at room temperature, the third instar larvae had a $17 \%$ frequency of ectopic endings on muscle fibers 7 and 6 . However, this level increased to $30 \%$ after undergoing the temperature shift regimen throughout development. Only after the mutants were shifted during both the embryo and the first instar was an increase in the frequency of ectopic endings above room temperature levels observed $(\sim 25 \%$ of hemisegments; Fig. 6). All other combinations of temperature shift during development produced larvae that continued to display room temperature levels of ectopic endings. This includes temperature shifts during embryogenesis, second and third instars or during the first, second, and third instars (Fig. 6). Therefore, temperature shifting during both late embryogenesis and the first instar is essential in promoting the temperature-enhanced sprouting effect.

\section{Collateral sprouting is mediated by the neuron}

To determine if sprouting is mediated by loss of action potentials in the motoneuron or by loss of postsynaptic potentials, both TTX and the orb web spider venom AR636 were used to block electrical activity in the embryo. TTX prevents action potentials by blocking $\mathrm{Na}$ channels only in the nerve, because Na conductances do not exist in Drosophila muscle fibers (Broadie and Bate, 1993d). In embryos, TTX has been shown to block action potentials and consequently EPSCs in the muscle fiber (Broadie and Bate, 1993c). The orb web spider toxin, AR636, prevents glutamate receptor activation (Jackson and Underwood, 1988). Glutamate receptors are found both in the CNS and at muscle fibers. Therefore, this treatment may not specifically block postsynaptic potentials. Previous studies indicate that embryonic treatment with AR636 prevents peristaltic movement as well as EPSCs after direct nerve stimulation (Broadie and Bate, 1993d). We have also shown that AR636 injection into stage 17 embryos abolishes peristaltic movement and it blocks postsynaptic potentials induced by glutamate iontophoresis in third instar larvae (data not shown; Jarecki et al., 1991). The toxins were applied for $4 \mathrm{hr}$ at $25^{\circ} \mathrm{C}$ from the time when growth cones first contact muscle targets to early stage 17 when differentiating motor endings are evident. Two experimental protocols, a filleted embryo culture system and the direct injection of toxins into intact embryos, were used.

As expected, TTX addition to the culture medium induced collateral sprouting onto the muscle fibers 6 and 7; as was observed in the activity mutants, embryonic sprouts always originate from the transverse nerve (Figs. 4B, $C$; 7; Table 3). However, we found that cultured embryos have a higher basal level of sprouting than intact embryos (Fig. 5), suggesting that the culture protocol promotes sprouting and NMJ development does not occur entirely normally. Even so, NMJ development proceeds as expected in culture as assessed by the normal temporal and spatial staining pattern of several neuronal and/or muscle markers, including fasciclins I, II, and III, HRP, and Toll (Fig. 7; Jan and Jan, 1982; Halpern et al., 1991; McAllister et al., 1992; Nose et al., 1992; Van Vactor et al., 1993). The addition of TTX to the culture bath increased the number of segments per embryo with a collateral sprout from $30 \%$ in control cultured embryos to $47 \%$ ( $p<0.005$ ). In contrast, AR636 treatment did not induce collateral sprouting (Table 3 ).

Since cultured embryos showed a higher-than-normal baseline of collateral sprouting, the experiment was repeated on a smaller 


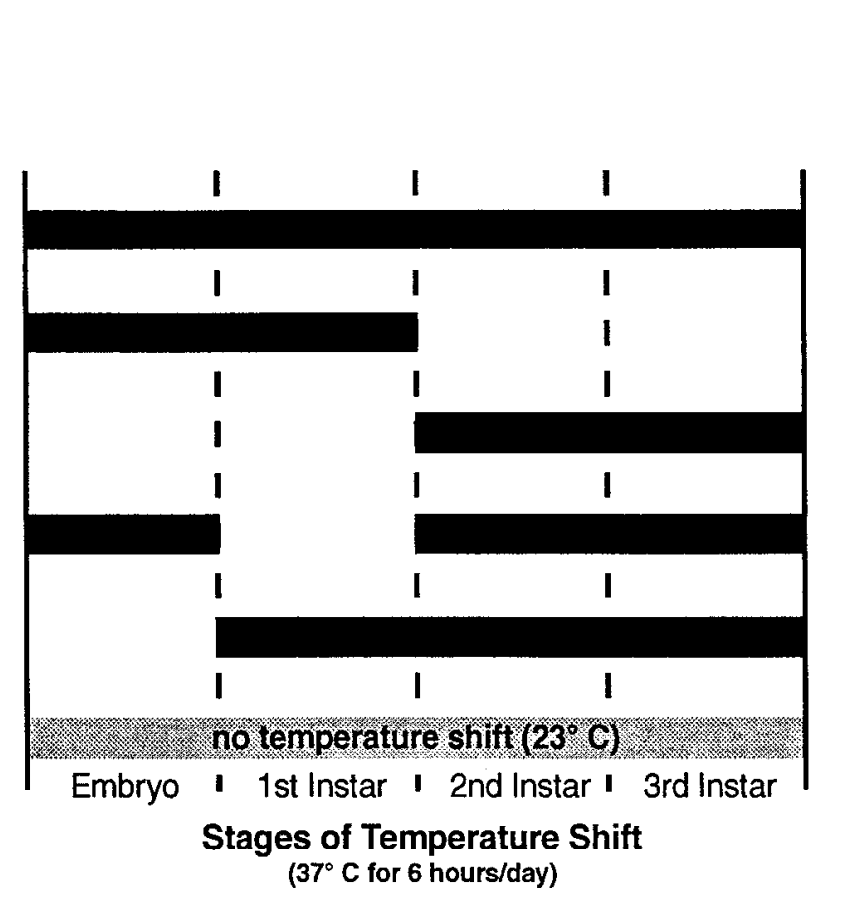

scale using a more difficult but more physiological procedure. After direct injection of the toxins into intact embryos, the same correlation was obtained as with the culture protocol (Figs. $4 C$, 8 ; Table 3). The frequency of collateral sprouting increased from $13 \%$ of hemisegments/animal in saline-injected embryos to $32 \%$ after TTX injection $(p<0.005)$. Both the control and experimental frequencies resulting from injection are very similar to those observed in wild-type and mutant embryos. As was seen in the culture experiments, AR636 did not affect collateral sprouting when injected into intact embryos (Table 3; Fig. 8). Therefore, it appears that blocking neural activity plays the major role in inducing collateral sprouting.

To test if loss of transmitter release is responsible for promoting collateral sprouting, third instar larvae bearing synaptotagmin mutations were analyzed. Synaptotagmin is a synaptic vesicle protein involved in neurotransmitter release. The generation of action potentials can still occur in these mutants, but the reduced neurotransmitter release leads to smaller postsynaptic potentials (Diantonio et al., 1993; Littleton et al., 1993). The AD1/T41 synaptotagmin transheterozygote was examined because it survives through larval development, but has a $75 \%$ reduction in evoked postsynaptic potentials in third instar larvae (Littleton et al., 1994). The frequency of collateral sprouting on

Table 3. Collateral sprouting in toxin treated embryos

\begin{tabular}{lll} 
Treatment & $\begin{array}{l}\text { \% Hemisegments/embryo } \\
\text { with a collateral sprout }\end{array}$ \\
\hline Culture & Control & $30 \pm 4(22,185)$ \\
& TTX & $47 \pm 4(25,225)^{*}$ \\
Injection & AR636 & $31 \pm 3(25,191)$ \\
& Saline & $13 \pm 5(13,95)$ \\
& TTX & $32 \pm 4(12,94)^{*}$ \\
& AR636 & $13 \pm 3(7,50)$
\end{tabular}

"Average \pm SEM, $n$ (animals, hemisegments).

* Significantly greater than control ( $p<0.005$ by Student's $t$ test). muscle fibers 6 and 7 did not increase over wild-type levels in third instar synaptotagmin larvae $(6.5 \%$ of hemisegments/animal, $n=167$ hemisegments in 18 animals). This supports the pharmacological evidence that the induction of collateral sprouting involves reductions in motoneuron activity as opposed to reductions in postsynaptic potentials.

\section{Discussion}

The Drosophila NMJ is characterized by precise synaptic connectivity. Our experiments demonstrate that this connectivity is regulated by neural activity during a critical period that extends from late embryogenesis through the first larval instar. Reducing neural activity both genetically and pharmacologically promotes inappropriate neuromuscular synapses. These are observed at multiple levels of activity block that range from not influencing the viability or behavior of the animals to the total loss of action potentials. They are first observed as collateral sprouts during embryogenesis and later during the third instar as ectopically placed motoneuron endings. These data reveal a novel mechanism that regulates synaptic connectivity in Drosophila.

\section{Collateral sprouting correlates with reductions in neural activity}

Our analysis demonstrates that reducing neural activity results in collateral sprouting at the Drosophila NMJ. All of the temperature-sensitive mutants that affect $\mathrm{Na}$ channels display at least a partial sprouting phenotype that is indistinguishable from the effects of TTX treatment. Each mutant shows a similar temperature-enhanced increase in collateral sprouting, whereas wildtype strains do not. Moreover, a duplication of the paralytic gene, which rescues nap ${ }^{t s}$ paralysis, also suppresses ectopic ending induction. Since double mutant combinations have higher levels of ectopic endings than single mutants, the frequency of sprouting appears to increase as neural activity decreases. Furthermore, when constructing the double mutants, the mutantbearing chromosome was brought in trans to a new combination of chromosomes, controlling for background effects. All of this 

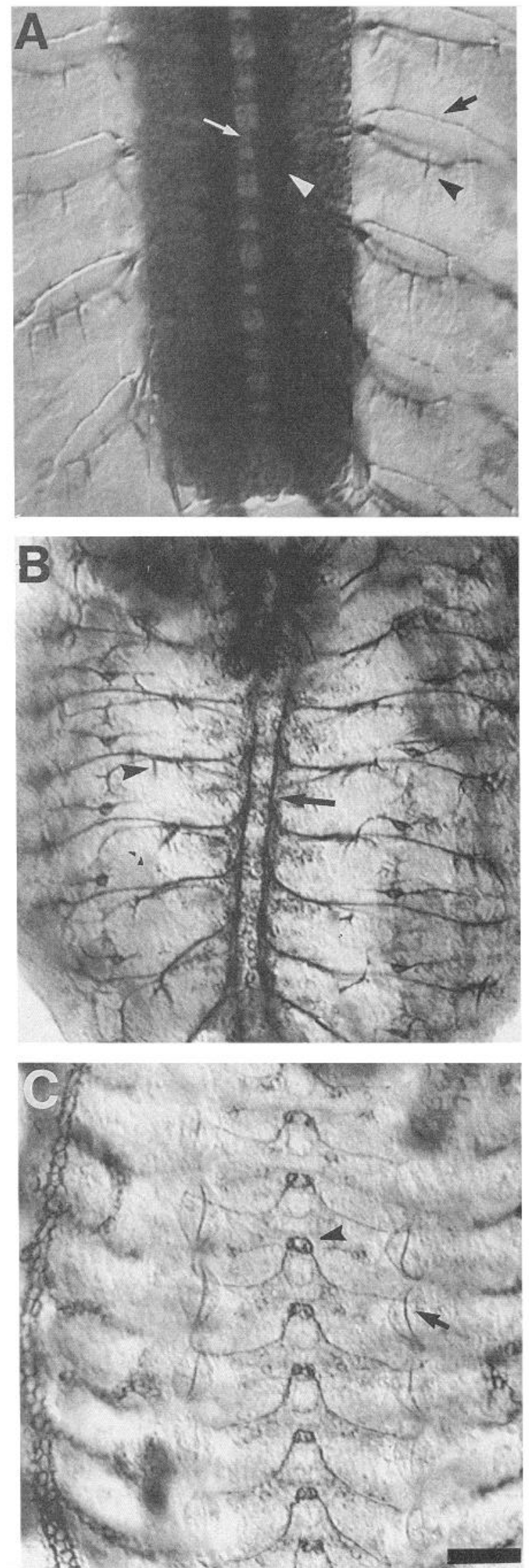

Figure 7. CNS development, motor ending development, and expression of several neuron-specific proteins is normal in embryos cultured

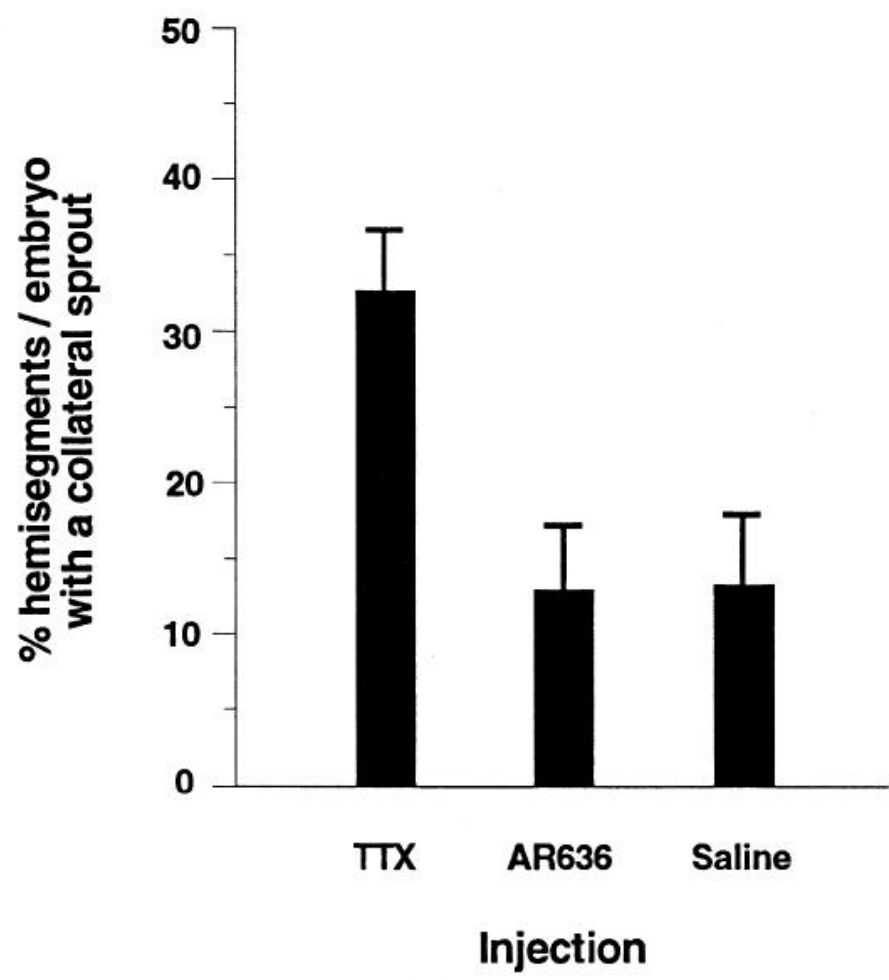

Figure 8. TTX injection into intact embryos increases collateral sprouting from the transverse nerve onto muscle fibers $7(p<0.005$ by $t$ test). AR636 injection does not influence the frequency of collateral sprouting.

data analyzed in concert indicates that genetic background effects or other functions of these proteins cannot account for this phenotype.

The embryonic sprouting phenotype correlates with the previously reported severity in the block of neural activity at the permissive and restrictive temperatures. For example, nap ${ }^{t s}$, tipE, and $s e i^{l}$ mutants have multiple permissive temperature phenotypes, including reduced $\mathrm{Na}$ channel numbers, TTX sensitivity, increased refractory period, and suppression of the eag sh leg shaking phenotype (Wu and Ganetzky, 1978, 1980; Ganetzky and Wu, 1982a,b; Jackson et al., 1984, 1985, 1986). Consistent with this, mutant embryos also have increased levels of sprouting at both permissive and restrictive temperatures. In contrast, para $^{t s l}$ larvae do not have any of the above-mentioned permissive temperature phenotypes, and even the null para ${ }^{k .5}$ embryos

$\leftarrow$

during stage 16 . Embryos were filleted at late stage 15/early stage 16, when the growth cones first contact muscle targets. They were cultured for $4 \mathrm{hr}$ at $25^{\circ} \mathrm{C}$ in L15 medium (see Materials and Methods). Anterior is up and the ventral midline is at the center. $A$, Cultured embryo stained with antibodies against horseradish peroxidase. Cultured embryos have normal CNS staining with anterior and posterior commissures (white arrow) and longitudinal connectives (white arrowhead). All nerve branches are labeled, including the transverse nerve (black arrow), and the developing motoneuron ending at the shared cleft of muscle fibers 6 and 7 (black arrowhead). B, Fasciclin II is expressed on multiple fascicles in the longitudinal connectives (black arrow), as well as on all nerve branches and innervation sites, including the $\mathrm{SNb}$ branch that innervates the shared cleft of muscle fibers 6 and 7 (black arrowhead). $C$, Fasciclin III is expressed normally on the RP motoneurons (black arrowheads), the axons that innervate muscle fibers 6 and 7 (black arrowhead), as well as the cleft between muscle fibers 6 and 7 (black arrow). Scale bar: $25 \mu \mathrm{m}$ for $A, 40 \mu \mathrm{m}$ for $B$ and $C$. 
remain active at room temperature (Keshishian et al., 1993). Accordingly, the para embryos do not exhibit the sprouting phenotype at permissive temperatures (Siddiqi and Benzer, 1976; Wu and Ganetzky, 1980; Kauvar, 1982; Ganetzky and Wu, 1986). However, at the restrictive temperature, action potentials are blocked in para embryos (Broadie and Bate, 1993a), and we observe elevated levels of sprouting at the NMJ. Consistent with these results, glutamate receptor localization remains normal in para $^{\text {tsl }}$ and para null embryos but is blocked at restrictive temperature (Broadie and Bate, 1993a).

Moreover, heterozygotic para ${ }^{l k 5}$ embryos reared at the restrictive temperature also show increases in collateral sprouting. Consistent with this, para $^{l k 5}$ has several described dominant phenotypes, including dominant lethality in a nap $p^{t s}$ background (Ganetzky, 1984) and dominant suppression of Shaker leg shaking behavior (Stern et al., 1990). Since paralks behaves as a null mutant, heterozygotes may have a greater reduction in the number of functional Na channels than the hypomorphic para $a^{t s l} \mathrm{em}-$ bryos. The temperature sensitivity of these mutants is proposed to be induced by reduced $\mathrm{Na}$ channel number as opposed to instability of the channel at elevated temperatures. This is because in Drosophila action potentials are blocked at elevated temperatures in animals with fewer $\mathrm{Na}$ channels because as temperature increases, the rate of potassium channel activation also increases, preventing sufficient depolarization of the nerve (Nelson and Wyman, 1989).

The critical period for ectopic ending formation includes both embryogenesis and the first instar

Critical period analysis indicates that reductions in neural activity are required during both cmbryogenesis and the first instar to promote ectopic ending formation. The formation of these ectopic endings appears to occur in two steps. Blocking neural activity during embryogenesis induces filopodial-like sprouting, whereas continued reduction into the first instar stabilizes the sprouts into differentiated synapses. This theory is supported by data obtained from the para ${ }^{t s t}$ mutant. para $^{\text {sst }}$ embryos raised at $34^{\circ} \mathrm{C}$, but not at $18^{\circ} \mathrm{C}$, have sprouts. These later disappear, and increases in ectopic neuromuscular synapses are never observed in para $^{\text {tsl }}$ larvae. Therefore, while collateral sprouts can be induced in paratsl embryos, they are not stabilized during subsequent development. In $n a p^{s s}, s e i^{l}$, and tipE embryos, collateral sprouting is observed at all temperatures. These mutant larvae have several permissive temperature phenotypes not observed in para $^{\text {ts }}$ larvae, including reductions in $\mathrm{Na}$ channel number in ligand binding assays, changes in refractory period and TTX sensitivity, and the ability to suppress eag sh leg shaking (Siddiqi and Benzer, 1976; Wu and Ganetzky, 1978, 1980; Ganetzky and Wu, 1982a,b; Kauvar, 1982; Jackson et al., 1984, 1985, 1986). Therefore, at permissive temperature $\operatorname{para}^{t s l}$ larvae appear to have a less severe hypoactivity phenotype than the other mutants. Our data suggests that an embryonic permissive-temperature sprouting phenotype predicts the appearance of ectopic endings in the third instar. This indicates that neural activity must be partially reduced throughout late embryogenesis and the first instar to promote stable ectopic endings, and that reducing neural activity just $6 \mathrm{hr} / \mathrm{d}$ during the temperature shift as in para $^{\text {tsI }}$ is not sufficient to induce ectopic ending formation. Therefore, ectopic ending formation seems to occur during two distinct phases during embryogenesis and the first instar.

The developmental time course for the growth of ectopic endings is similar to that of the native endings. Embryonic neuro- muscular endings are immature with most ending differentiation occurring during larval development (Keshishian et al., 1993). Consistent with this, embryonic collateral sprouts are always filopodial. In addition, all arise from the transverse nerve, unlike third instars in which they arise from a variety of nerve sources. This suggests that ectopic endings from the other sources develop later during the last stages of embryogenesis. This is consistent with the critical period data, which suggests that neural activity must be reduced during stage 17 of embryogenesis, which for technical reasons is later than the period when embryonic sprouting was assessed. Therefore, it appears that collateral sprouts are induced during embryogenesis but differentiate during larval development. This proposed two-step model for the induction of sprouting follows the known developmental time course of the native endings suggesting that the induction of ectopic ending formation in Drosophila occurs during early periods when connectivity is normally being established.

\section{Mechanisms of paralysis-induced collateral sprouting}

Several mechanisms may account for the induction of collateral sprouting at the Drosophila NMJ. Our data provide strong evidence that the induction of collateral sprouting results from the loss of electrical activity in the neuron as opposed to the muscle. For example, the spider toxin AR636, which is a potent blocker of glutamatergic PSPs, and synaptotagmin mutants, which reduce neurotransmitter release, do not result in sprouting, whereas a series of Na channel mutants and TTX do. The results obtained from the seizure mutants also point to a mechanism in which electrical activity is required in the motoneuron as opposed to the muscle fiber. seizure is a member of the eag potassium channel family with a hyperactive phenotype at the adult giant fiber (Kasbekar et al., 1987; L. Hall, personal communication). However, only $s e i^{I}$, not $s e i^{2}$, displays a reduced number of $\mathrm{Na}$ channels and Na current density, and we only observed elevated levels of ectopic endings in $s e i^{l}$ mutants, suggesting that collateral sprouting is induced by the Na channel defect in the neuron. All of the above evidence suggests that the loss of electrical activity in the motoneuron rather than the muscle fiber is responsible for the induction of collateral sprouting. This is in contrast to the vertebrate NMJ, where blocking acetylcholine receptor activation is sufficient to induce collateral sprouting (Brown, 1984).

However, the involvement of the muscle fiber in the induction of collateral sprouting cannot be ruled out. Drosophila motoneurons express several cotransmitters that may continue to stimulate the muscle fiber after glutamate receptor activation is prevented (Anderson et al., 1988; Cantera and Nässel, 1992; Gorczyca et al., 1993; Keshishian et al., 1993; Zhong and Pena, 1995). However, the synaptotagmin protein probably regulates the release of all neurotransmitters, as it is expressed at both dense and clear vesicles (Walch-Solimena et al., 1993). Alternatively, the loss of neural activity might indirectly induce a change in the muscle fiber that would in turn cause motoneuron growth. In this case, the requirement for electrical activity would be autonomous to the motor neuron, but the muscle fiber would be involved in the induction of sprouting.

Although repulsive signals have been suggested to regulate connectivity (Nose et al., 1994; Matthes et al., 1995), it is unlikely that native endings actively repel their neighbors. For example, muscle fibers are multiply innervated (Broadie and Bate, 1993c) and can accept target-deprived foreign inputs, which argues against the secretion of a repulsive factor from the native ending (Cash et al., 1992). Therefore, we favor an inductive 
mechanism rather than a repulsive one for the induction of collateral sprouting.

In vertebrate systems, blocking postsynaptic activity induces collateral sprouting, and the secretion of growth factors from muscle fibers has been implicated in this process. For example, denervation or paralysis cause the reexpression of insulin-like growth factors I and II in adult muscle fibers, although these growth factors are nomally down-regulated during synapse elimination (Ishii, 1989; Caroni and Schneider, 1994). Moreover, they can promote collateral sprouting in vivo (Caroni and Grandes, 1990; Caroni and Schneider, 1994). Synaptic activity regulates the expression of a number of growth-related molecules at the vertebrate NMJ including IGF, NCAM, GAP-43, and myogenin, suggesting that synaptic activity may serve as a developmental switch after synaptogenesis (Couvalt and Sanes, 1985; Reiger et al., 1985; Ishii, 1989; Caroni and Grandes, 1990; Eftimei et al., 1991; Caroni and Becker, 1992; Caroni and Schneider, 1994). Perhaps the initiation of synaptic activity terminates a growth program after synaptogenesis, and blocking synaptic activity later in life reinitiates it. However, it remains unclear whether a similar mechanism occurs in Drosophila.

\section{The role of electrical activity in NMJ development}

In Drosophila, blocking electrical activity does not affect axon pathfinding or target selection (Broadie and Bate, 1993a). Therefore, as in many vertebrate systems, these early events are independent of neural activity. In vertebrates, subsequent processes such as synapse elimination do depend on synaptic activity (reviewed by Coleman and Lichtman, 1993). However, electrophysiological evidence as well as the fact that muscle fibers remain polyinnervated throughout larval development suggests that competition does not occur in Drosophila (Broadie and Bate, 1993c; Keshishian et al., 1993). Consistent with this, muscle fibers can accept innervation by a neighboring target-deprived motoneuron without altering the morphology of the native synapse (Cash et al., 1992).

Later in Drosophila NMJ development, after outgrowth and target selection are complete, reducing neural activity disrupts NMJ connectivity. Collateral sprouting in Drosophila can also be induced by denervation (Keshishian et al., 1993, 1994; Halfon et al., 1995). As in paralysis, denervation-induced sprouts first appear in late embryogenesis, just after the completion of target selection. Furthermore, they localize glutamate receptors (Broadie and Bate, 1993a, 1993b) and develop into functional ectopic endings originating from the same nerve sources as those induced by paralysis (Keshishian et al., 1993, 1994; Halfon et al., 1995). However, it remains unclear if the mechanisms responsible for sprouting are the same in paralysis and denervation in Drosophila. For example, denervation causes a frequency of ectopic endings on muscle fibers 6 and 7 that is twofold higher than the highest frequencies ever observed in larvae with reductions in neural activity. Moreover, denervation probably involves signals from the muscle fiber, and paralysis-induced sprouting appears to involve the loss of neural activity. Therefore, the presence of the nerve, not synaptic transmission, may prevent growth-promoting signals from the muscle fibers.

Electrical activity also regulates motor ending morphogenesis in Drosophila. For example, the double mutant eag sh, which increases nerve excitability, leads to more elaborate larval motor endings in third instar larvae (Budnik and Gorczyca, 1992). Several other experiments indicate that electrical activity regulates morphogenesis even earlier in development. Altered activity lev- cls have been shown to change both the number of branches at the NMJ as well as the total terminal length by the first instar (Broadie and Bate, 1993a; Keshishian et al., 1993). Many of these changes are likely to occur postembryonically, since the most branches and boutons arise during larval life (Keshishian et al., 1993).

Our experiments demonstrate a novel aspect of the regulation of neuromuscular connectivity. Although the Drosophila NMJ is characterized by precise synaptic connections, this connectivity can be modified by changes in the levels of neural activity. Early events like nerve outgrowth and target selection are activity independent, but subsequent processes, such as the maintenance of precise innervation, depend on neural activity. The well-characterized Drosophila neuromuscular system, with its added benefit of genetic manipulation, is perfectly suited for further experimentation that will lead to a greater understanding of the molecular mechanisms underlying plasticity in all organisms.

\section{References}

Anderson MS, Halpern ME, Keshishian H (1988) Identification of the neuropeptide transmitter proctolin in Drosophila larvae: characterization of muscle fiber-specific neuromuscular endings. J Neurosci $8: 242-255$.

Atwood HL, Govind CK, Wu C-F (1993) Differential ultrastructure of synaptic terminals on the ventral longitudinal abdominal muscles in Drosophila larvae. J Neurobiol 24:1008-1024.

Balice-Gordon RJ, Lichtman JW (1993) In vivo observations of preand postsynaptic changes during the transition from multiple to single innervation at the developing neuromuscular junction. J Neurosci 13: $834-855$.

Bate M (1990) The embryonic development of larval muscle fibers in Drosophila. Development 110:791-804.

Bellen HJ, O'Kane CJ, Wilson C, Grossniklaus U, Pearson RK, Gehring WJ (1989) P-element mediated enhancer detection: a versatile method to study development in Drosophila. Genes Dev 3:1288-1300.

Broadie K, Bate M (1993a) Activity-dependent development of the neuromuscular synapse during Drosophila embryogenesis. Neuron $11: 607-619$

Broadie K, Bate M (1993b) Innervation directs receptor synthesis and localization in Drosophila embryo synaptogenesis. Nature 361:350353.

Broadie KS, Bate M (1993c) Development of the embryonic neuromuscular synapse of Drosophila melanogaster. J Neurosci 13:144166.

Broadie KS, Bate M (1993d) Development of larval muscle properties in the embryonic myotubes of Drosophila melanogaster. J Neurosci 13:167-180.

Broadie KS, Skaer H, Bate M (1992) Whole-embryo culture of Drosophila: development of embryonic tissues in vitro. Rouxs Arch Dev Biol 201:364-375.

Brown MC (1984) Sprouting of motor nerves in adult muscles: a recapitulation of ontogeny. Trends Neurosci 7:10-14.

Brown MC, Ironton R (1977) Motor sprouting induced by prolonged tetrodotoxin block of nerve action potential. Nature 265:459-461.

Budnik V, Gorczyca M (1992) SSB, an antigen, that selectively labels morphologically distinct synaptic boutons at the Drosophila larval neuromuscular junction. J Neurobiol 23:1054-1066.

Budnik V, Zhong Y, Wu C-F (1990) Morphological plasticity of motor axons in Drosophila mutants with altered excitability. J Neurosci 10: $3754-3768$.

Cantera R, Nässel DR (1992) Segmental peptidergic innervation of abdominal targets in larval and adult dipteran insects revealed with an antiserum against leukokinin I. Cell Tiss Res 269:459-471.

Caroni P, Becker M (1992) The downregulation of growth-associated proteins in motoneurons at the onset of synapse elimination is controlled by muscle activity and IGF1. J Neurosci 123:3849-3861.

Caroni P, Grandes P (1990) Nerve sprouting in innervated adult skeletal muscle induced by exposure to elevated levels of insulin-like growth factors. J Cell Biol 110:1307-1317.

Caroni P, Schneider C (1994) Signaling by insulin-like growth factors 
in paralyzed skeletal muscle: rapid induction of IGF1 in muscle fibers and prevention of interstitial cell proliferation by IGF-BP5 and IGFBF4. J Neurosci 14:3378-3388.

Cash S, Chiba A, Keshishian H (1992) Alternate neuromuscular target selection following the loss of single muscle fibers in Drosophila. J Neurosci 12:2051-2064.

Cline HT, Constantine-Paton M (1989) NMDA receptor antogonists disrupt the retinotectal topographic map. Neuron 3:412-426.

Coleman H, Lichtman JW (1993) Interactions between nerve and muscle: synapse elimination at the developing neuromuscular junction. Dev Biol 156:1-10.

Couvalt J, Sanes JR (1985) Neural cell adhesion molecule (N-CAM) accumulates in denervated and paralyzed skeletal muscles. Proc Nat Acad Sci USA 82:4544-4558.

Diantonio A, Parfitt KD, Schwarz TL (1993) Synaptic transmission persists in synaptotagmin mutants of Drosophila. Cell 73:1281-1290.

Eftimie R, Brenner HR, Bounanno A (1991) Myogenin and MyoD join a family of skeletal muscle genes regulated by electrical activity. Proc Nat Acad Sci USA 88:1349-1353.

Feng G, Deak P, Chopra M, Hall LM (1995) Cloning and functional analysis of TipE a novel membrane protein that enhances Drosophila para sodium channel function. Cell 82:1001-1011.

Ganetzky B (1984) Genetic studies of membrane excitability in Drosophila: lethal interactions between two temperature-sensitive paralytic mutants. Genetics 108:879-911.

Ganetzky B (1986) Neurogenetic analysis of Drosophila mutations affecting sodium channels Synergistic effects on viability and nerve conduction in double mutants involving tipE. J Neurogenet 3:19-31.

Ganetzky B, Wu C F (1982a) Drosophila mutants with opposing effects on nerve excitability: genetic and spatial interaction in repetitive firing. J Neurophysiol 7:501-514.

Ganetzky B, Wu C F (1982b) Indirect suppression involving behavioral mutants with altered excitability in Drosophila melanogaster. Genetics 100:597-614

Ganetzky B, Wu C F (1983) Neurogenetic analysis of potassium currents in Drosophila: synergistic effects on neuromuscular transmission in double mutants. J Neurogenet 1:17-28.

Ganetzky B, Wu C F (1986) Neurogenetics of membrane excitability in Drosophila. Annu Rev Genet 20:13-44.

Goodman CS, Shatz CJ (1993) Developmental mechanisms that generate precise patterns of neuronal connectivity. Cell [Suppl] 72:7798.

Gorczyca MG, Augart C, Budnik V (1993) Insulin-like receptor and insulin-like peptide are localized at neuromuscular junctions in Drosophila. J Neurosci 13:3692-3704.

Gorczyca M, Phillis RW, Budnik V (1994) The role of tinman, a mesodermal cell fate gene, in axon pathfinding during development of the transverse nerve in Drosophila. Development 120:2143-2152.

Halfon MS, Hashimoto C, Keshishian H (1995) The Drosophila Toll gene functions zygotically and is necessary for proper motoneuron and muscle development. Dev Biol 169:151-167.

Halpern ME, Chiba A, Johansen J, Keshishian H (1991) Growth cone behavior underlying the development of stereotypic synaptic connections in Drosophila embryos. J Neurosci 11:3227-3238.

Holland RL, Brown MC (1980) Postsynaptic transmission block can cause terminal sprouting of a motor nerve. Science 207:649-651.

Hong CS, Ganetzky B (1994) Spatial and temporal expression patterns of two sodium channel genes in Drosophila. J Neurosci 14:51605169.

Ishii DN (1989) Relationship of insulin-like growth factor II gene expression in muscle to synaptogenesis. Proc Natl Acad USA 86:28982902.

Jackson FR, Wilson SD Strichartz, GR Hall, LM (1984) Two types of mutants affecting voltage-sensitive sodium channels in Drosophila melanogaster. Nature 308:189-191.

Jackson FR, Gitschier J, Strichartz GR, Hall LM (1985) Genetic modification of voltage-sensitive sodium channels in Drosophila: gene dosage studies of the seizure locus. J Neurosci 5:1144-1151.

Jackson FR, Wilson SD, Hall LM (1986) The tipE mutation of Drosophila decreases saxitoxin binding and interacts with other mutations affecting nerve membrane excitability. J Neurogenet 3:1-17.

Jackson H, Underwood P (1988) Spider toxins as tools for dissecting elements of excitatory amino acid transmission. Trends Neurosci 11: $278-283$.
Jan LY, Jan YN (1976a) Properties of the larval ncuromuscular junction in Drosophila melanogaster. J Physiol (Lond) 262:189-214.

Jan LY, Jan YN (1976b) L-Glutamate as excitatory transmitter at the Drosophila larval neuromuscular junction. J Physiol (Lond) 262:215236.

Jan LY, Jan YN (1982) Antibodies to horseradish peroxidase as specific neuronal markers in Drosophila and grasshopper embryos. Pro Natl Acad Sci USA 79:2700-2704

Jarecki JE, Sun Y-A, Anderson MDS (1991) The role of neural activity in establishing neuromuscular ending morphology during Drosophila embryogenesis. Soc Neurosci Abstr 18:580.

Johansen J, Halpern ME, Johansen KM, Keshishian H (1989a) Stereotypic morphology of glutamatergic synapses on identified muscle cells of Drosophila larvae. J Neurosci 9:710-725.

Johlansen J, Halpen ME, Keshishian H (1989b) Axonal guidance and the development of muscle fiber-specific innervation in Drosophile embryos. J Neurosci 9:4318-4332.

Kasbekar DP, Nelson JC, Hall LM (1987) Enhancer of seizure: a new genetic locus in Drosophila melanogaster defined by interaction with temperature-sensitive paralytic mutations. Genetics 116:423-431.

Kauvar LM (1982) Reduced $\left[{ }^{3} \mathrm{H}\right]$-tetrodotoxin binding in the $n a p^{2.5}$ paralytic mutant of Drosophila. Mol Gen Genet 187:172-173.

Kernan MJ, Kuroda MI, Kreber R, Baker BS, Ganetzky B (1991) nap $p^{t s,}$ a mutation affecting sodium channel activity in Drosophila, is an allele of mle, a regulator of $\mathrm{X}$ chromosome transcription. Cell 66 : 949-959.

Keshishian H, Chiba A, Chang TN, Halfon M, Harkins EW, Jarecki J, Wang LS, Anderson MD, Cash S, Halpern ME, Johansen J (1993) Cellular mechanisms governing synaptic development in Drosophila melanogaster. J Neurobiol 24:757-787.

Keshishian H, Chang TN, Jarecki J (1994) Precision and plasticity during Drosophila neuromuscular development. FASEB J 8:731-737.

Kulkarni SJ, Padhye A (1982) Temperature-sensitive paralytic mutations on the second and third chromosomes of Drosophila melanogaster. Genet Res 40:191-199.

Kuroda MI, Kernan MJ, Kreber R, Ganetzky B, Baker BS (1991) The maleless protein associates with the $\mathrm{X}$ chromosome to regulate dosage compensation in Drosophila. Cell 66:935-947.

Littleton JT, Bellen HJ, Perin MS (1993) Expression of synaptotagmin in Drosophila reveals transport and localization of synaptic vesicles to the synapse. Development 118:1077-1088.

Littleton JT, Stern M, Perin M, Bellen HJ (1994) Calcium dependence of neurotransmitter release and rate of spontaneous vesicle fusions are altered in Drosophila synaptotagmin mutants. Proc Nat Acad Sci USA 91:10888-10892.

Loughney K, Krebe R, Ganetzky B (1989) Molecular analysis of the para locus, a sodium channel gene in Drosophila. Cell 58:11431154.

Matthes DJ, Sink H, Kolodkin AL, Goodman CS (1995) Semaphorin II can function as a selective inhibitor of specific synaptic arborizations. Cell 81:631-639.

McAllister L, Goodman CS, Zinn K (1992) Dynamic expression of the cell adhesion molecule fasciclin I during embryonic development in Drosophila. Development 115:267-276.

Meyer RL (1982) Tetrototoxin blocks the formation of ocular dominance columns in the goldfish. Science 218:589-591.

Nelson JC, Wyman RJ (1989) Examination of paralysis in Drosophila temperature-sensitive paralytic mutations affecting sodium channels: a proposed mechanism of paralysis. J Neurobiol 21:453-469.

Nose A, Mahajan VB, Goodman CS (1992) Connectin: a homophilic cell adhesion molecule on a subset of muscles and motoneurons that innervate them in Drosophila. Cell 70:553-567.

Nose A, Tacheichi M, Goodman CS (1994) Ectopic expression of connectin reveals a repulsive function during growth cone guidance and synapse formation. Neuron 13:525-539.

O'Brien RD, Ostberg AC, Vrbova G (1978) Observations on the elimination of polyneuronal innervation in developing mammalian skeletal muscle. J Physiol (Lond) 282:571-582.

O'Dowd DK, Aldrich RW (1988) Voltage-clamp analysis of sodium channels in the wild-type and mutant Drosophila neurons. J Neurosci $8: 3633-3643$.

O'Dowd DK, Germeraad SE, Aldrich RW (1989) Alterations in the expression and gating of Drosophila sodium channels by mutations in the para gene. Neuron 2:1301-1311.

Patel NH, Snow PM, Goodman CS (1987) Characterization and clon- 
ing of fasciclin III: a glycoprotein expressed on a subset of neurons and axon pathways in Drosophila. Cell 48:975-988.

Reh TA, Constantine-Paton M (1985) Eye-specific segregation requires neural activity in threc-cycd Rana pipiens. J Ncurosci 5:1132-43.

Reiger F Grumet M, Elelman GM (1985) N-CAM at the vertebrate neuromuscular junction. J Cell Biol 101:285-293.

Salkoff L Butler A, Wei A, Scavarda N Giffen K, Ifune C, Goodman R, Mandel G (1987) Genomic organization and deduced amino acid sequence of a putative sodium channel gene in Drosophila. Science 237:744-749.

Schmidt JL, Edwards DL (1983) Activity sharpens the map during the regeneration of the retinotectal projection in goldfish. Brain Res 209: $29-39$.

Siddiqi O, Benzer S (1976) Neurophysiological defects in temperaturesensitive mutants of Drosophila melanogaster. Proc Natl Acad Sci USA 73:3253-3257.

Sink H, Whitington PM (1991a) Location and connectivity of abdominal motoneurons in the embryo and larva of Drosophila melanogaster. J Neurobiol 22:298-311.

Sink H, Whitington PM (1991b) Pathfinding in the central nervous system and periphery by identified embryonic Drosophila motor axons. Development 112:307-316.

Snow PM, Bieber AJ, Goodman CS (1989) Fasciclin III: a novel homophilic adhesion molecule in Drosophila. Cell 59:313-323.

Stern M, Kreber R, Ganetzky B (1990) Dosage effects of a Drosophila sodium channel gene on behavior and axonal excitability. Genetics 124:133-143.

Stryker MP, Harris WA (1986) Binocular impulse blockade prevents the formation of ocular dominance in cat visual cortex. $J$ Neurosci $6: 2117-2133$.
Suzuki DT, Grigliatti T, Williamson R (1971) Temperature-sensitive mutants in Drosophila melanogaster: a mutation ( para $^{t s l}$ ) causing reversible adult paralysis. Proc Natl Acad Sci USA 68:890-893.

Thompson W (1983) Synapse elimination in neonatal rat muscle is sensitive to pattern of muscle use. Nature 302:614 616 .

Van Vactor D, Sink H, Fambrough D, Tsoo R, Goodman CS (1993) Genes that control neuromuscular specificity in Drosophila. Cell 73: 1137-1153.

Walch-Solimena C, Takei K, Marek KL, Midyett K, Sudhof TC, De Camilli P, Jahn R (1993) Synaptotagmin: a membrane constituent of neuropeptide-containing large dense-core vesicles. J Neurosci 13: $3895-3903$.

Wang J, Renger J, Griffith LC, Greenspan RJ, Wu CF (1995) Concomitant alterations of physiological and developmental plasticity in Drosophila CaM kinase II-inhibited synapses. Neuron 13:1373 1384.

Woodard C, Huang T, Sun H, Helfand SL, Carlson J (1989) Genetic analysis of olfactory behavior in Drosophila: a new screen yields the ota mutants. Genetics 123:315-326.

Wu C-F, Ganetzky B (1980) Genetic alteration of nerve membrane excitability in temperate-sensitive paralytic mutants of Drosophila melanogaster. Nature 286:814-816.

Wu C-F, Ganetzky B, Jan YN, Jan LY, Benzer S (1978) A Drosophila mutant with a temperature sensitive block in nerve conduction. Proc Nat Acad Sci USA 75:4047-4051.

Zhong Y, Pena LA (1995) A novel synaptic transmission mediated by a PACAP-like neuropeptide in Drosophila. Neuron 14:527.

Zhong Y, Budnik V, Wu C-F (1992) Synaptic plasticity in Drosophila memory and hyperexcitable mutants: role of cAMP cascade. J Neurosci 12:644-651. 